
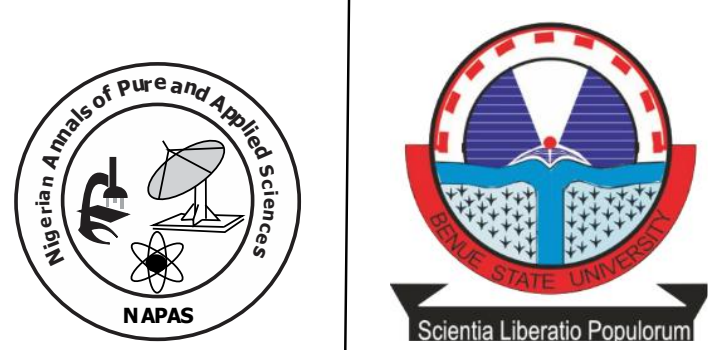

\title{
Assessment of Radiofrequency Radiation Exposure Levels from Mobile Phones And Wireless Hotspots in Some Parts of Makurdi-Nigeria
}

\author{
${ }^{1}$ Akaagerger, N.B., ${ }^{2}$ Kaki, D.K., ${ }^{3 P h i l i p, ~ A . l ., ~}{ }^{4}$ lkyo, B.A. \\ ${ }_{1}^{1}$ Department of Physics, Benue State University, Makurdi, Nigeria \\ bnguvan@gmail.com \\ 2Department of Physics, Benue State University, Makurdi, Nigeria \\ dkaki77@gmail.com \\ 3 Department of Physics, Akawe Torkula College of Advanced \& \\ Professional Studies, Makurdi, Nigeria \\ ${ }^{4}$ Department of Physics, Benue State University, Makurdi, Nigeria. \\ biky012@yahoo.com
}

\begin{abstract}
Radio waves and Microwaves also known as Radio Frequency Radiations (RFR) have been associated with health hazards. This research was carried out to assess the safety level of these radiations in Makurdi, Benue State. Measurement of RFR power density and electric field strength were carried out for mobile phones. A Cornet 15SA Electrosmog meter was used for assessing RFR exposure levels. Measurements of RFR power density $\left(\mathrm{mWm}^{-2}\right)$ and electric field strength $\left(\mathrm{Vm}^{-1}\right)$ for wireless hotspots were carried out within $10 \mathrm{~m}$ radius to offices and open areas with high occupancy. Radiation levels from mobile phones were measured on the surface with mobile phones in contact with the meter in a normal direction such that maximum readings are obtained. A survey questionnaire was also designed to assess the level of knowledge of the residents concerning the effects of exposure to RF radiations. The field strength and Power density from mobile phones were found to range from $0.1946 \mathrm{Vm}^{-1}$ to $26.2446 \mathrm{Vm}^{-1}$ and $0.1004 \mathrm{mWm}^{-2}$ to $1.827 \mathrm{Wm}^{-2}$ respectively. These are within ICNIRP recommended value of $10 \mathrm{Wm}^{-2}$ for members of the public. Environmental levels of RFR in areas with RFR generating devices showed that RFR levels ranged from $5.0595 \mathrm{Vm}^{-1}\left(0.0679 \mathrm{Wm}^{-1}\right)$ to $7.3961 \mathrm{Vm}^{-1}\left(0.145 \mathrm{Wm}^{1}\right)$. Also, assessment of areas with Wi-Fi hotspots showed that the presence of hotspots significantly increased background levels of RFR. These values were compared with measurements carried out in areas without RF devices which ranged from $0.10212 \mathrm{Vm}^{-1}$ $\left(0.02766 \mathrm{mWm}^{-2}\right)$ to $1.7334 \mathrm{Vm}^{-1}\left(0.0079 \mathrm{Wm}^{-2}\right)$. The presence of devices was seen to increase the amount of radiation in the environment. The questionnaire results indicated that there was need to further educate the public on the effects of these radiations and precautionary to be adopted for safety.
\end{abstract}

Keywords: Radiofrequency radiation, Power density, Field strength, Mobile Phones, Wireless hotspot, WiFi Makurdi, Nigeria 


\section{Introduction}

Mobile phones have experienced a boom in Nigeria since introduction in 2001. This is evidenced by the availability of these gadgets (Forencher et al, 2019). Wireless hotspots have also been introduced at several institutions for easy access to the internet. However, this increase comes with the potential for increased exposure to radio and microwaves which are the means by which these devices operate.

The International Commission on NonIonizing Radiation Protection (ICNIRP) has developed safety standards for exposure to radio and microwaves (ICNIRP, 2020). Several studies have assessed levels of Radiofrequency radiations. For example, the effects of radiofrequency radiation on human Ferritin (a macromolecule for the long term iron storage function in human serum and plasma) was carried out (Fattahi et al., 2012). Samples were found to be rapidly depleted of Ferritin after thirty minutes of exposure to radiofrequency radiation $(900 \mathrm{MHz})$ from cell phones. Similarly, the effects of radiofrequency radiation $(1800 \mathrm{MHz})$ on embryonic neural stem cells responsible for brain development showed that radiofrequency radiation impaired outgrowth of the cells (Chen et al., 2014). It was suggested that more attention be given to the potential adverse effects of radiofrequency exposure on brain development. Another study conducted in 2009 assessed 1100 GSM handsets of different makes and models in Lagos. Power density values obtained ranged from 1.294 to $16.83 \mathrm{Wm}^{-2}$. The research concluded that the population was at risk because these values were above limits set by ICNIRP (Aweda et al., 2009). Again, another study (Eyinna and Idonijie, 2016) assessed the radiofrequency radiation and noise impacts of base stations positioned in residential areas at Ntueke. SAR values obtained ranged from 6.71 to $603.87 \mu \mathrm{Wkg}^{-1}$ which were said to be higher than ICNIRP limits of exposure. Results from these studies and show how necessary it is to monitor and ensure that exposure levels are kept within safety limits as recommended by local and international regulatory bodies.

\section{Theoretical Consideration}

Radiofrequency Radiations are electromagnetic waves produced by electromagnetic oscillation circuits. They are plane polarized with their plane of polarization being determined by circuit geometry. Polarized electromagnetic waves (in contrast to no-polarized) can produce interference effects and induce coherent forced-vibrations on charged or polar molecules within a medium.

When a polarized, non-ionizing electromagnetic wave passes through a mass of polar or charged molecules, such as those composing biological tissue, it induces a forced-oscillation on each of these particles that it meets and transfers to each of them a tiny part of its energy. This induced oscillation will be most intense on the free particles which carry a net electric charge such as the free (mobile) ions that exist in large concentrations in all types of cells or extracellular biological tissue determining practically all cellular or biological functions (Panagopoulos \& Margaritis, 2013). The induced oscillation will be much weaker or even totally negligible on the polar biological macromolecules and the water molecules that do not have a net charge and additionally are usually bound chemically to other molecules.

After each such event of interaction between the wave and a charged or polar particle, the remaining wave continues on its way through the tissue possibly scattered by a tiny angle and reduced by a tiny amount in its amplitude and intensity. After large numbers of such events, depending on the tissue's density, and the number of polar or charged molecules, the remaining wave, if any, leaves the tissue as a scattered wave of reduced amplitude or intensity.

As the intensity, E of radiofrequency waves decreases after interaction with charged or polar molecules of a medium, its energy density $\mathrm{W}_{\mathrm{em}}$ decreases as well. The energy density, $W_{\text {em }}$ of a plane wave is given by Panagopoulous (2013) as;

$W_{e m}=\varepsilon_{0} E^{2}$ 
$W_{e m}$ is the total energy per unit volume of the electromagnetic wave, and $E$ the intensity of the electric component of the wave $\epsilon_{0}$ is permittivity of vacuum $\varepsilon_{o}=8.854 \times 10^{-12}$ $\mathrm{C}^{2} / \mathrm{N} \cdot \mathrm{m}^{2}$ is the vacuum permittivity. That means that a part of its energy per unit volume is transferred to the charged orpolar molecules of the medium.

Specific absorption rate is a measure of how transmitted radiofrequency energy is absorbed by human tissue. SAR is measured in watts per kilogram $(\mathrm{W} / \mathrm{kg})$. Absorption is a function of tissue permittivity and conductivity, and frequency of radiator. Safe limits for human exposure are sometimes based on whole body exposure averaged over 0.1 hours (6 minutes).

Specific Absorption Rate can be calculated using the mathematical relation given in equation 2 below (ICNIRP, 2020).

$S A R=\frac{\sigma \times E^{2}}{\rho}$

Where $\sigma$ is the conductivity $(\mathrm{S} / \mathrm{m}), \mathrm{E}$ is the Root Mean Square (RMS) electric field $\left(\mathrm{Vm}^{-1}\right)$ and $\rho$ is the density.

The incident power of a radiofrequency field is also given by (Sanders, 2001):

$S=\frac{E^{2}}{377}$

The SAR values have not been calculated in this study due to complications involved in measurement. Results have been presented in terms of Power density and field strength which quantify external exposure levels.

\section{Materials and Methods}

Structured questionnaire and measurements were used for the study. The questionnaire was designed to assess the knowledge of RF exposure and how often respondents made use of RFR emitting devices. Two hundred copies of the questionnaire were used. An Electrosmog meter manufactured by CORNET company USA (Model number:
ED15SA) with build-in Spectrum Analyser for $2.4 \mathrm{GHz}$ band was used in carrying out Power Density and Electric Field Strength measurements on mobile phones and wireless hotspots.. The device is designed to carry out broadband measurement of radiations in radiofrequency range. It is a tool for quick measurement of High frequency (radiofrequency) Radio wave field strength $(100 \mathrm{MHz}-3 \mathrm{GHz})$, power density, and frequency spectrum of $2.4 \mathrm{GHz}$ band wireless applications.

One hundred and twenty five (125) different models and makes of phones were sampled from different regions of the study area and the amount of power density and field strength measured. Measurements of power density and electric field strength from mobile phones were carried. The electrosmog meter was made to be in contact with mobile phones such that the meter was in a normal direction when maximum readings are obtained.

Also, measurements were conducted for power density and electric field strength from wireless (Wi-Fi) hotspots at various locations within Benue State University. These measurements were conducted to assess the background level of RF radiation due to the presence of wireless hotspots. In addition to this, some areas within the study area with high number of radiofrequency radiation generating devices were assessed for background levels of radiation. These locations were designated as $\mathrm{A}, \mathrm{B}, \mathrm{C}$, and $\mathrm{D}$ respectively. The values obtained were compared with background levels of RFR in areas without RFR sources.

\section{Results and Discussion}

The results obtained from the study are presented in this section. The results for the amount of radiation in areas with high number radiofrequency radiation emitting devices is shown in Table I while that emitted by Wi-Fi hotspots is presented in table II. A summary of results for mobile phones showing the maximum and minimum values obtained for each brand is presented in Table III. 
Table 1: Results for radiation in areas with high number of radiofrequency radiation emitting devices

\begin{tabular}{cccc}
\hline S/N & LOCATION & $\begin{array}{l}\text { POWER DENSITY } \\
\left(\mathbf{m W M}^{-2}\right)\end{array}$ & FIELD STRENGTH $\left(\mathbf{V M}^{-1}\right)$ \\
\hline 1. & $\mathrm{~A}$ & 145.1 & 7.3961 \\
2. & $\mathrm{~B}$ & 20.04 & 2.7487 \\
3. & $\mathrm{C}$ & 230.0 & 9.3139 \\
4. & $\mathrm{D}$ & 67.9 & 5.0595 \\
\hline
\end{tabular}

Table 2: Results for radiofrequency emissions from wi-fi hotspots

\begin{tabular}{cllc}
\hline S/N & LOCATION & $\begin{array}{l}\text { POWER DENSITY } \\
\left(\mathbf{m W m}^{-2}\right)\end{array}$ & $\begin{array}{l}\text { FIELD STRENGTH } \\
\left(\mathbf{V m}^{-1}\right)\end{array}$ \\
\hline 1 & Spot 1 & 0.01264 & 0.90719 \\
2 & Spot 2 & 0.02766 & 0.10212 \\
3 & Spot 3 & 0.09500 & 0.18925 \\
4 & Spot 4 & 0.06050 & 0.15103 \\
5 & Spot 5 & 0.02964 & 0.10571 \\
6 & Spot 6 & 0.33260 & 0.35411 \\
7 & Spot 7 & 0.03907 & 0.12137 \\
8 & Spot 8 & 0.27000 & 0.31905 \\
9 & Spot 9 & 0.21970 & 0.28780 \\
10 & Spot 10 & 0.56800 & 0.46275 \\
11 & Spot 11 & 1.38600 & 0.72286 \\
12 & Spot 12 & 0.36470 & 0.37080 \\
13 & Spot 13 & 7.97000 & 1.73340 \\
14 & Spot 14 & 0.3033 & 0.33815 \\
15 & Spot 15 & 0.1027 & 0.19677 \\
16 & Spot 16 & 0.0981 & 0.19231 \\
\hline
\end{tabular}

Table 3: Results for radiofrequency emissions from mobile phones

\begin{tabular}{|c|c|c|}
\hline PHONE BRAND & $\begin{array}{l}\text { P.D } \\
\left(\mathbf{m W m}^{-2}\right)\end{array}$ & $\begin{array}{l}\text { FS (E) } \\
\left(\mathbf{V m}^{-1}\right)\end{array}$ \\
\hline Itel & $\begin{array}{l}{ }^{\mathrm{a}} 0.1004 \\
{ }^{\mathrm{b}} 1250.700\end{array}$ & $\begin{array}{l}{ }^{\mathrm{a}} 0.1946 \\
{ }^{\mathrm{b}} 21.7144\end{array}$ \\
\hline Infinix & $\begin{array}{l}{ }^{\mathrm{a}} 0.10040 \\
\mathrm{~b} 202.1000\end{array}$ & $\begin{array}{l}\text { a } 0.1946 \\
\text { b8.7289 }\end{array}$ \\
\hline Gionee & $\begin{array}{l}{ }^{\mathrm{a}} 0.2301 \\
\mathrm{~b} 459.1000\end{array}$ & $\begin{array}{l}\text { a9.3138 } \\
\text { b13.1560 }\end{array}$ \\
\hline Samsung & $\begin{array}{l}{ }^{\mathrm{a}} 0.22480 \\
{ }^{\mathrm{b}} 1827.0000\end{array}$ & $\begin{array}{l}{ }^{\mathrm{a}} 0.2911 \\
\mathrm{~b} 26.2446\end{array}$ \\
\hline Huawei & $\begin{array}{l}\text { a } 761.3000 \\
\text { b } 1520.000\end{array}$ & $\begin{array}{l}{ }^{\mathrm{a}} 16.9414 \\
\mathrm{~b} 23.9383\end{array}$ \\
\hline Nokia & $\begin{array}{l}{ }^{\mathrm{a}} 0.29500 \\
\mathrm{~b} 1153.0000\end{array}$ & $\begin{array}{l}{ }^{\mathrm{a}} 0.3335 \\
{ }^{\mathrm{b}} 20.8490\end{array}$ \\
\hline
\end{tabular}


The readings at offices and other locations due to emissions from Wi-Fi hotspots as shown in Table I were found to range from $0.10571 \mathrm{Vm}^{-}$ $1\left(0.02964 \mathrm{mWm}^{-2}\right)$ to $1.7334 \mathrm{Vm}^{-1}(7.97000$ $\left.\mathrm{mWm}^{-2}\right)$. Results for measurements in areas with high density of RF device users are shown in Table I. Results in Tables I and II showed that the density of devices increased the amount of radiofrequency radiation considerably. This is observed from the higher levels of radiation at Spot 13 (Table II) with power density of $1.7334 \mathrm{Vm}^{-2}\left(4.362 \times 10^{-3} \mathrm{Jkg}^{-}\right.$ $\left.{ }^{1}\right)$ as compared with others in the table. The values at Spot 13 were significantly increased by the presence of a large number of users of the hotspot at the time when the readings were taken. It will therefore be important to limit the number of devices in a small location or to provide enough space in order to reduce radiation levels.

The power density $\left(\mathrm{mWm}^{-2}\right)$ and electric field Strength $\left(\mathrm{Vm}^{-1}\right)$ obtained for Itel, Infinix, Gionee, Samsung, Huawei, and Nokia brands of mobile phones are as presented in Table III above. All RF emissions were found to be within the ICNIRP (2020) limit of $42 \mathrm{Vm}^{-}$ $1\left(10 \mathrm{Wm}^{-2}\right)$ with Samsung brand having the highest exposure values $\left(26.2446 \mathrm{Vm}^{-1}, 1.827\right.$ $\mathrm{Wm}^{-2}$ ). These results are in agreement with other studies which found that results were in within safety limits. (Iortile \& Agba, 2014; Humbe, 2010; Shalangwa, 2010). It is however observed to be in variance with results of Aweda et al (2009) who found that results were non-compliant. This variation might be said to be attributed to improvement in device technology since 2009. Other countries however, have set stricter measures. Italy $(0.1$ $\left.\mathrm{Wm}^{-2}\right)$, Swiss $\left(0.04 \mathrm{Wm}^{-2}\right)$ and Russia (0.02 $\mathrm{Wm}^{-2}$ ). The readings compared with these standards are found to have exceeded the range.

The results obtained from the questionnaire showed that, fifty six percent $(56 \%)$ of the respondents had at least one radiation emitting device, twenty four and a half percent $(24.5 \%)$ of them had two devices while nineteen and a half percent $(19.5 \%)$ had three devices and only three $(1.5 \%)$ had no device. The average number of device per respondent was 1.64 with the average duration of use being four to six hours in a day. Furthermore, one hundred and thirty nine $(72.4 \%)$ of the respondents indicated that they were aware of the dangers of radiation emitting devices. Despite this knowledge, only twenty two $(11.6 \%)$ of the respondents indicated that they checked the radiation specification of the devices before purchase. One hundred and fifty two $(80 \%)$ of the respondents said they did not check the specifications while sixteen $(8.4 \%)$ thought it was not necessary to check these specifications. The respondents were also seen to have additional sources of exposure besides these devices. Sixty seven point two percent $(67.2 \%)$ of the respondents indicated they had stationary sources of RFR around their homes or places of work. Thirty $(16.1 \%)$ had two sources while ten $(5.2 \%)$ had three sources around them. These results show that there is a potential for combined exposure to be high due to these additional sources. There is a need to ascertain the distances at which these sources are located from the homes and places of work due to high occupancy time in these places.

\section{Conclusion}

The Radiofrequency Radiation exposure level of different nodels of six major brands of mobile phones (Itel, Infinix. Huawei, Gionee, Samsung and Nokia) were measured. These were found to range from $0.1946 \mathrm{Vm}^{-1}$ $\left(0.1004 \mathrm{Wm}^{-2}\right)$ to $26.2446\left(1.8270 \mathrm{Wm}^{-2}\right)$. This is within the safety limit of emission from mobile phones provided by International Commission of Non-Ionizing Radiation protection (ICNIRP, 2020). Hence, exposures are considered to be safe. The emissions created by the wireless hotspots were seen to increase the amount of radiation present within the surrounding buildings as compared with areas without devices. It is therefore necessary to employ measures that help in limiting the number of RFR emitting devices and to maintain a safe distance from residential areas and buildings with high occupancy in order to reduce the level of exposure and ensure that people and the environment are not unduly exposed. Also, there is need to educate the 
public on the effects of exposure to radiofrequency radiations and measures that should be employed to ensure safety.

\section{References}

Aweda, M. A., Ajekighe, A. T., Ibitoye, A. Z., Evwhierhurhoma, B. O., \& Eletu, O. B. (2009). Potential Health Risks due to Telecommunications Radiofrequency Radiation Exposures in Lagos State Nigeria. Nig Qj HospMed.; 19(1)6-14.

Chen C., Ma, Q., Deng P., Zhu, G., Zhang, L., He, M., Yonghui, L., Duan, W., Pei, M., Yu, Z., \& Zhou, Z. (2014). Exposure to $1800 \mathrm{MHz}$ Radio Frequency Radiation Impairs Neurite Outgrowth of Embryonic Neural Stem cells. Scientific Reports 4, Article number 5103.

Eyinna, I. P., \& Idonjie M. (2016). Radiofrequency Radiation (RFR) and Noise Impacts of Positioning Base Station within Residential Areas in Ntueke, Nigeria. Frontiers in Science, 6(2):31-35.

Fattahi-Asl, J., Baradaran-Ghahfarokhi, M., Karbalae, M., Baradaran-Ghahfarokhi M., \& Baradaran-Ghahfarokhi, H. R. (2012). Effects of radiofrequency Radiation on Human Ferritin: An Invitro Enzymum Assay. J Med Signals Sens.; 2(4): 235-240.

Forencher I., Husnjak S., Cvitic I. and Jovovic (2019). Determinants of Mobile Phone Owneership in Nigeria. Telecommunications Policy 43. https://doi.org/10.1016/j.telpol.2019.03 .001

International Commission on Non-Ionizing Radiation Protection, (ICNIRP), (2020). "Guidelines for limiting exposure to time-varying electric, magnetic, and electromagnetic fields (100kHz to $300 \mathrm{GHz})$ ", Health Physics, 118(5):483-524.

Iortile, J.T, Agba, E.H.(2014). Assessment of Radio Frequency Radiation Distribution Arround Mobile Base Stations in Makurdi, Benue State. International Journal of Natural Sciencies Research, 2(1): 1-4.

Panagopoulos, D. J. \& Margaritis, L. H, (2013). Theoretical Considerations for the Biological Effects of Electromagnetic Fields, In: Stavroulakis $P$, ed. "Biological Effects of Electromagnetic Fields”, Springer: 5-33.

Sanders F. (2001); "Conversion of Power Measured in a Circuit to Incident Field Strength and Incident Power Density, and Corrections to Measured Emission Spectra for Non-Constant Aperture Measurement Antennas". NTIA Report 01-383

Shalangwa, D. A. (2010). Measurement of Exposure of Radiofrequency Field (RF) Radiation from Global System for Mobile Communication (GSM) Masts. Journal of Electrical and Electronics Engineering Research Vol.2 (3), pp7584. 